\title{
Exterior Algebra and Projections of Polytopes
}

\author{
P. Filliman \\ Department of Mathematics, Western Washington University, Bellingham, WA 98225, USA
}

Communicated by G. D. Chakerian

\begin{abstract}
This paper explores the metrical properties of convex polytopes by means of the classical Plücker embedding of the Grassmannian $G(k, n)$ of $k$-planes in $R^{n}$ into the exterior algebra $\Lambda_{k} R^{n}$. The results follow from the description of the volume of the projection of a polytope into a $k$-plane by a piecewise linear function on $G(k, n)$. For example, the Hodge-star operator is used to obtain the volume of a polytope from its Gale transform. Also, the classification of the faces of $G(2, n)$ (or $G(n-2, n)$ ) imply that the largest projection within a particular combinatorial type is unique if $k=2$ or $n-2$.
\end{abstract}

\section{Introduction}

The exterior (or Grassmann) algebra is a beautiful classical theory which can be used to represent geometric properties of $R^{n}$ by algebraic operations. This method has had applications in many areas of geometry, one example being the recent use of chirotopes (or oriented matroids) to investigate the combinatorial properties of polytopes (see [2] and [3]). This paper uses the exterior algebra to gain insight into the metric properties of polytopes, in particular, the volume of their projections.

The first section contains a review of the properties of the exterior algebra, especially in light of the connection with the geometry of $R^{n}$ mentioned above. The principal object of study is the Grassmann manifold $G(k, n)$ of all $k$ dimensional subspaces of $R^{n}$. Let $Q$ be a convex polytope in $R^{n}$ and let $V(Q: L)$ be the $k$-dimensional volume of the orthogonal projection of $Q$ into a $k$-plane $L \in G(k, n)$. The key to the results of this paper is

Theorem 1. The function $V(Q: L)$ is piecewise linear on $G(k, n)$.

Although this result is not new (see p. 25 of [5]), the proof in Section 2 contains 
an alternative construction of $V(Q: L)$ which yields some interesting new corollaries.

One example concerns the Gale, c.s., and zonal transforms. These transforms have proved quite useful in obtaining combinatorial results for polytopes (see [23], [26], and [30]). As an application of Theorem 1, we prove in Section 3 that the transform of a polytope also gives its volume.

A special case of the above occurs when $Q$ is an $n$-dimensional simplex. In this case, the decomposition of $G(k, n)$ induced by $V(Q: L)$ corresponds to combinatorial equivalence of polytopes with at most $n+1$ (labeled) vertices. This decomposition refines to the decomposition induced by chirotopes.

The final sections deal with the problem of maximizing $V(Q: L), L \in G(k, n)$. Section 4 relates the largest projections of the regular simplex, cross polytope, and cube to the solutions of other extremum problems and gives some necessary conditions. One interesting result is that if the vertices of a maximal projection of $T^{n-1}$ lie on a sphere, then they also solve the problem of finding the largest volume of the convex hull of $n$ points on a sphere in $R^{k}$. Some bounds and solutions for the projections of a cube can be found in [6], [11], and [19].

Section 5 contains a uniqueness result for the problem above:

Theorem 11. There is at most one maximal projection in each open region of $G(k, n), k=2$ or $n-2$, on which $V(Q: L)$ is linear.

The proof establishes a connection between this problem and the use of "calibrations" in the study of area-minimizing surfaces (see [28]).

\section{Exterior Algebra}

The definitions and notation will mostly follow Federer's book [9]. The Grassmann manifold $G(k, n)$ of $k$-dimensional linear subspaces of $R^{n}$ is to be topologized as follows:

A $k$-frame $X=\left\{x_{1}, \ldots, x_{k}\right\}$ is a set of $k$ linearly independent vectors of $R^{n}$. We denote by $X$ the $k \times n$ matrix whose $i$ th row consists of the coordinates of $x_{i}$. The collection of all $k$-frames forms an open subset of $R^{k n}$, called the Stiefel manifold $V(k, n)$. Define a map

$$
\begin{aligned}
q: V(k, n) & \rightarrow G(k, n), \\
X & \rightarrow L=\operatorname{lin} X,
\end{aligned}
$$

which takes each $k$-frame $X$ to the $k$-plane $L$ which it spans, and give $G(k, n)$ the quotient topology under this map.

Lemma. The Grassmann manifold $G(k, n)$ is a smooth, compact manifold of dimension $k(n-k)$. The correspondence $L \rightarrow L^{\perp}$, which assigns to each $k$-plane its orthogonal $(n-k)$-plane, defines a diffeomorphism between $G(k, n)$ and $G(n-k, n)$ (see 5.1 of $[27])$. 
The twofold cover of $G(k, n)$ is the set of oriented $k$-planes $\tilde{G}(k, n)$. These manifolds can be defined alternatively as the homogeneous spaces $O(n) /(O(k) \times$ $O(n-k))$ and $S O(n) /(S O(k) \times S O(n-k))$, respectively [32, Vol. 5, pp. 403, 414]

The exterior algebra $\Lambda_{*} R^{n}=\oplus \Lambda_{k} R^{n}$ is the quotient $\otimes_{*} R^{n} / 1$, where $\otimes_{*} R^{n}$ denotes the tensor algebra of $R^{n}$ and $I$ denotes the ideal generated by $\{x \otimes x \mid x \in$ $R^{n}$ \}. It follows that multiplication is skew-symmetric, i.e., if $x, y \in R^{n}$, then $x \wedge y=-y \wedge x$.

The elements of $\Lambda_{k} R^{n}$ are called " $k$-vectors." Given a basis $\left\{e_{1}, \ldots, e_{n}\right\}$ of $R^{n}$, the $k$-vectors

$$
e_{\lambda}=e_{\lambda_{1}} \wedge \cdots \wedge e_{\lambda_{k}}, \quad \lambda \in \Lambda(n, k)
$$

where

$$
\Lambda(n, k)=\left\{\left(\lambda_{1}, \ldots, \lambda_{k}\right) \in N^{k} \mid 1 \leq \lambda_{1}<\cdots<\lambda_{k} \leq n\right\}
$$

form a basis for $\Lambda_{k} R^{n}$ as a vector space of dimension $M=\left(\begin{array}{l}n \\ k\end{array}\right)$.

There is a natural embedding of $G(k, n)$ into real projective space $P^{M-1}$, called the Plücker embedding. First define

$$
\text { i: } \begin{aligned}
V(k, n) & \rightarrow \Lambda_{k} R^{n}, \\
X & \rightarrow x_{1} \wedge \cdots \wedge x_{k} .
\end{aligned}
$$

If $f: L \rightarrow L, L=\operatorname{lin} X$, is a linear map, then $i(f(X))=\left(f\left(x_{1}\right)\right) \wedge \cdots \wedge\left(f\left(x_{k}\right)\right)=$ (det $f) x_{1} \wedge \cdots \wedge x_{k}$. Hence, the image under $i$ of all the $k$-frames in $L$ is a line through the origin in $\Lambda_{k} R^{n}$. It follows that $i$ can be extended to a map which embeds $G(k, n)$ as a submanifold of $P^{M-1}$ cut out by quadratic polynomials [14, p. 209].

Below is a list of some basic properties of $G(k, n)$ and $\Lambda_{k} R^{n}$ (see Chapter 1 of [9]):

1. Given a linear map $f: R^{n} \rightarrow R^{n}$, we define the $k$ th-compound map $\Lambda_{k} f: \Lambda_{k} R^{n} \rightarrow \Lambda_{k} R^{n}$ by letting

$$
\left(\Lambda_{k} f\right)\left(e_{\lambda}\right)=f\left(e_{\lambda_{1}}\right) \wedge \cdots \wedge f\left(e_{\lambda_{k}}\right), \quad \lambda \in \Lambda(n, k),
$$

and then extending by linearity to all of $\Lambda_{k} R^{n}$. The classical Cauchy-Binét theorem implies

$$
\Lambda_{k}(f \circ g)=\left(\Lambda_{k} f\right) \circ\left(\Lambda_{k} g\right)
$$

2. If $\left\{e_{1}, \ldots, e_{n}\right\}$ is an orthonormal basis of $R^{n}$, then a $k$-vector $\xi \in \Lambda_{k} R^{n}$ can be expressed as

$$
\xi=\sum \xi_{\lambda} e_{\lambda} .
$$


The numbers $\xi_{\lambda}, \lambda \in \Lambda(n, k)$, are known as the Plücker coordinates of $\xi$, and if $\xi=i(X)$, they are identical to the $(k \times k)$-subdeterminants of $X$.

The usual Euclidean inner product on $\Lambda_{k} R^{n}$ is given by

$$
\langle\xi, \eta\rangle=\sum \xi_{\lambda} \eta_{\lambda}
$$

$\xi, \eta \in \Lambda_{k} R^{n}$, and

$$
|\xi|=\sqrt{\langle\xi, \xi\rangle}
$$

is the norm of $\xi$. Note that $\left\{e_{\lambda} \mid \lambda \in \Lambda(n, k)\right\}$ forms an orthonormal basis of $\Lambda_{k} R^{n}$. Formula (1.2) can be used to show that if $f$ is an orthogonal transformation of $R^{n}, \Lambda_{k} f$ is also an orthogonal transformation of $\Lambda_{k} R^{n}$. Hence, in the proof of many of the statements below, it suffices to take $\xi=e_{1} \wedge \cdots \wedge e_{k}$.

This inner product has a useful geometric interpretation. Let $X, Y \in V(k, n)$, $\xi=i(X)$, and $\eta=i(Y)$. Then

$$
\langle\xi, \eta\rangle=\operatorname{det}\left(\mathbf{X} \mathbf{Y}^{\prime}\right)= \pm|\xi| V(Y: X)
$$

where $V(Y: X)$ equals the volume of the parallelotope spanned by the orthogonal projection $Y^{\prime}$ of $Y$ into $\operatorname{lin} X$. Assuming $|\xi|=1,(1.4)$ implies

$$
\frac{1}{k !}|\langle\xi, \eta\rangle|=V\left(\operatorname{conv}\left\{0, Y^{\prime}\right\}\right.
$$

This formula is the basis of the representation of the volume of polytopes in the next section.

3. Interior multiplication is the bilinear map

$$
\rfloor: \Lambda_{p} R^{n} \times \Lambda_{q} R^{n} \rightarrow \Lambda_{q-p} R^{n}
$$

$p \leq q$, defined by

$$
\langle\xi \wedge \eta, \Phi\rangle=\langle\xi, \eta\rfloor \Phi\rangle,
$$

$\xi \in \Lambda_{q-p} R^{n}, \eta \in \Lambda_{p} R^{n}$, and $\Phi \in \Lambda_{q} R^{n}$. If $\mu \in N^{k}$, we define sgn $\mu$ to be the sign of the permutation of $\mu$ which puts its elements in increasing order. It follows from (1.6) that if $\lambda \in \Lambda(n, p)$ and $\mu \in \Lambda(n, q)$,

$$
\left.e_{\lambda}\right\rfloor e_{\mu}= \begin{cases}0 & \text { if } \lambda \notin \mu, \\ \operatorname{sgn}(\nu, \lambda) e_{\nu} & \text { if } \mu=\nu \cup \lambda,\end{cases}
$$

$\nu \in \Lambda(n, q-p)\left(e_{\varnothing}=1\right)$. The formula for combining interior and exterior multiplication is

$$
(\xi \wedge \eta)\rfloor \Phi=\xi\rfloor(\eta\rfloor \Phi),
$$


$\xi \in \Lambda_{p} R^{n}, \eta \in \Lambda_{q} R^{n}, \Phi \in \Lambda_{r} R^{n}$, and $p+q \leq r$. They can also be used to define a linear isomorphism *: $\Lambda_{k} R^{n} \rightarrow \Lambda_{n-k} R^{n}$ called the Hodge-star operator. Given an orthonormal basis $\left\{e_{1}, \ldots, e_{n}\right\}$ of $R^{n}$, and $\xi \in \Lambda_{k} R^{n}$, let

$$
* \xi=\xi\rfloor\left(e_{1} \wedge \cdots \wedge e_{n}\right)
$$

The equation

$$
\langle\xi, \Phi\rangle=\left\langle{ }^{*} \xi{ }^{*} \Phi\right\rangle
$$

$\xi$, $\Phi \in \Lambda_{k} R^{n}$, will be quite important in the section on transforms.

4. A $k$-vector is said to be "simple" if it is the exterior product of $k$ vectors of $R^{n}$, i.e., if it belongs to $i(V(k, n))$. If $\xi \neq 0$ is a simple $k$-vector, we will let $L(\xi)$ denote the $k$-dimensional subspace associated to $\xi$. As noted above, two simple vectors $\xi$ and $\eta$ represent the same subspace if and only if $\xi=c \eta, c$ a nonzero scalar. This also implies that the simple, unit $k$-vectors can be identified with the oriented $k$-planes $\tilde{G}(k, n)$.

The following relations are valid for simple vectors $\xi \in \Lambda_{p} R^{n}$ and $\eta \in \Lambda_{q} R^{n}$, $p \leq q($ see $[2]):$

$$
\begin{aligned}
L\left(^{*} \xi\right) & =L(\xi)^{\perp} \quad \text { and } \quad|* \xi|=|\xi|, \\
L(\xi \wedge \eta) & =L(\xi) \oplus L(\eta) \quad \text { if } \quad L(\xi) \cap L(\eta)=0, \\
L(\xi\rfloor \eta) & \left.=L(\xi)^{\perp} \cap L(\eta) \quad \text { and } \quad \mid \xi\right\rfloor \eta|=| \eta \mid V(X: Y),
\end{aligned}
$$

where $\xi=i(X)$ and $\eta=i(Y)$.

Finally, the quadratic relations which indicate when a $k$-vector $\xi$ is simple can be written as

$$
(\eta\rfloor \xi) \wedge \xi=0, \quad \forall \eta \in \Lambda_{k-1} R^{n},
$$

or

$$
(\xi\rfloor \Phi)\rfloor \xi=0, \quad \forall \Phi \in \Lambda_{k+1} R^{n}
$$

(see [12]).

\section{Projection Form}

Let $Q \subset R^{n}$ be an $n$-dimensional convex polytope, and let $\Pi(Q: L)$ be the orthogonal projection of $Q$ into the oriented $k$-plane $L \in G(k, n)$. This section contains a proof that the $k$-dimensional volume $V(Q: L)=V(\Pi(Q: L))$ is a piecewise linear function on $G(k, n)$. We begin with some notation. 
The set of faces of $Q$ will be denoted by $F(Q)$ and the set of $k$-faces by $F_{k}(Q)$. Fix a labeling vert $Q=\left\{y_{1}, \ldots, y_{m}\right\}$ of the vertices of $Q$. This induces a corresponding labeling of the points $\Pi(\operatorname{vert}(Q): L)$, which can be used to define an equivalence relation on $G(k, n)$. Two $k$-planes $L$ and $L^{\prime}$ are said to be equivalent if the map between the polytopes $\Pi(Q: L)$ and $\Pi\left(Q: L^{\prime}\right)$ given by the labelings above is a combinatorial equivalence. The equivalence class of $L$ will be denoted by $\langle L\rangle$. Finally, the previous section implies that choosing an orientation of $L$ is equivalent to choosing a unit, simple $k$-vector $\xi$ with $L(\xi)=L$.

Theorem 1. The volume $V(Q: L), L \in G(k, n)$, can be represented by a function on $\Lambda_{k} R^{n}$ which is linear on each equivalence class $\langle L\rangle$. This representation is unique (up to sign) whenever $\langle L\rangle$ is open in $G(k, n)$.

Proof. Assume $0 \in$ int $Q$. Let $P=\Pi(Q: L), L \in G(k, n)$, and let $\xi \in \tilde{G}(k, n)$ be an orientation of $L$. We choose a triangulation $\Gamma(\partial P)$ of the boundary $\partial P$ of $P$ into $(k-1)$-simplices whose vertices belong to vert $Q$. This gives a decomposition of $P$ into disjoint pyramids, each having apex 0 and base in $\Gamma(\partial P)$. Given $\lambda \in \Lambda(m, k)$, we define

$$
\Phi_{\lambda}=\frac{1}{k !} \operatorname{sign}\left(\left\langle\xi, y_{\lambda}\right\rangle\right) y_{\lambda}
$$

where $y_{\lambda}=y_{\lambda_{1}} \wedge \cdots \wedge y_{\lambda_{k}}$. Formula (1.5) implies that $\left\langle\xi, \Phi_{\lambda}\right\rangle$ equals the volume of the pyramid conv $\left\{0, \Pi\left(F_{\lambda}: L\right), F_{\lambda}=\operatorname{conv}\left\{y_{\lambda_{1}}, \ldots, y_{\lambda k}\right\}\right.$. Hence

$$
V(Q: L)=\langle\xi, \Phi\rangle
$$

for

$$
\Phi=\left\{\Sigma \Phi_{\lambda} \mid \Pi\left(F_{\lambda}: L\right) \in \Gamma(\partial P)\right\}
$$

The construction of $\Phi$ depends on the combinatorial type of $P$, the labeling of vert $Q$, and a choice of orientation of $L$. This proves the first statement of the theorem.

The uniqueness of $\Phi$ follows from the fact that no neighborhood of $G(k, n)$ lies in a hyperplane of $\Lambda_{k} R^{n}[17$, Vol. 1, p. 310]. Thus if $\langle L\rangle$ is open, it must contain a basis of $\Lambda_{k} R^{n}$, and (2.2) gives the coordinates of $\Phi$ with respect to this basis.

The $k$-vector $\Phi$ in (2.2) will be called a "projection form" of $Q$. In order to avoid always referring to the $k$-plane $L$, we will sometimes write $\Pi(Q: \xi)$ for $\Pi(Q: L(\xi))$, and write $\chi(\Phi)$ for those $\xi \in \tilde{G}(k, n)$ such that $V(Q: \xi)=\langle\xi, \Phi\rangle$, i.e., $L(\chi(\Phi))=\langle L\rangle$.

It will be useful to give an alternative construction of $\Phi$ which does not assume $0 \in$ int $Q$. Let $F_{\mu}=$ conv $\left\{y_{\mu_{1}}, \ldots, y_{\mu_{k+1}}\right\}, \mu \in \Lambda(m, k+1)$, be a $k$-dimensional simplex with vertices belonging to vert $Q$. We can associate to $F_{\mu}$ a simple $k$-vector $f_{\mu}$ such that $L\left(f_{\mu}\right)$ is parallel to aff $F_{\mu}$ and $\left|f_{\mu}\right|=V\left(F_{\mu}\right)$. This determines $f_{\mu}$ up 
to a sign which represents an orientation of aff $F_{\mu}$. Choose a triangulation $\Gamma(P)$ of $\boldsymbol{P}$ into disjoint $k$-simplies, and define

$$
\Phi_{\mu}=\operatorname{sign}\left(\left(\xi, f_{\mu}\right)\right) f_{\mu}
$$

and

$$
\Phi=\left\{\Sigma \Phi_{\mu} / \Pi\left(F_{\mu}: \xi\right) \in \Gamma(P)\right\}
$$

This $\Phi$ also satisfies (2.2). A third method of constructing $\Phi$ can be found on pp. 24-25 of [5]. In [5] a projection $\Pi(Q: L)$ is said to be "sharp" if the region $\chi(\Phi)$ is open in $\tilde{G}(k, n)$.

It will also be necessary to have an algebraic description of $\chi(\Phi)$. That is,

$$
\begin{aligned}
\chi(\Phi)= & \left\{\eta \in \tilde{G}(k, n) \mid \operatorname{sign}\left(\left\langle\eta, f_{\mu}\right\rangle\right)=\operatorname{sign}\left(\left\langle\xi, f_{\mu}\right\rangle\right)\right. \\
& \text { if } \left.F_{\mu} \in F_{k}(Q) \text { and } \operatorname{dim}\left(F_{\mu} \cap \Pi_{\xi}^{-1}(\partial P)\right) \geq k-1\right\} .
\end{aligned}
$$

The set $\chi(\Phi)$ is bounded by hyperplane sections

$$
H_{\mu}=\left\{\eta \in \tilde{G}(k, n) \mid\left\langle\eta, f_{\mu}\right\rangle=0\right\} \text {. }
$$

Equations (2.5) and (2.7) imply that (2.2) holds also on the closure $\overline{\chi(\Phi)}$ of $\chi(\Phi)$ in $\tilde{G}(k, n)$.

A very important special case of the above occurs when $Q$ is an $n$-dimensional simplex. Then the partition of $G(k, n)$ given by the equivalence relation above corresponds to the combinatorial types of the convex hull of labeled sets of $n+1$ points which span $R^{k}$, and the sharp regions represent simplicial polytopes. The concept of chirotope (or oriented matroid) equivalence is a proper refinement of this relation in $\Lambda_{k} R^{n}$. This concept has been used to obtain many new results on the realizability of configurations [2], [3] and upper bounds for the number of combinatorial types [13].

For polytopes $Q \subset R^{n}$ with dimension $\operatorname{dim} Q<n$, we have

Proposition 2. The maximum of $V(Q: L)$ is attained by a $k$-plane $L$ parallel to aff $Q$.

Proof. If $\operatorname{dim} Q=n$ there is nothing to prove, so we assume $\operatorname{dim} Q<n$ and let $W$ be the subspace parallel to aff $Q$. Then any vector $x \in R^{n}$ has a unique representation

$$
x=x^{\prime}+x^{\prime \prime}
$$

with $x^{\prime} \in W$ and $x^{\prime \prime} \in W^{\perp}$.

Suppose $\xi=x_{1} \wedge \cdots \wedge x_{k} \in \tilde{G}(k, n)$ and $L(\xi) \notin W$. The remarks following Theorem 1 imply

$$
V(Q: \xi)=\langle\xi, \Phi\rangle
$$


where $\Phi=\sum \Phi_{\mu}$ is given by (2.5). Now, if $\eta$ is any simple vector with $L(\eta) \cap W^{\perp} \neq$ 0 , then

$$
\left\langle\eta, f_{\mu}\right\rangle=0
$$

since $L\left(f_{\mu}\right) \subset W($ see (1.4)). It follows immediately that

$$
\left\langle\xi, f_{\mu}\right\rangle=\left\langle\xi^{\prime}, f_{\mu}\right\rangle
$$

where $\xi^{\prime}=x_{1}^{\prime} \wedge \cdots \wedge x_{k}^{\prime}$. Substituting (2.8) in (2.6), we see that $\Pi\left(Q: \xi^{\prime}\right)$ has the same combinatorial type as $\Pi(Q: \xi)$, and therefore $\Phi$ is also a projection form of $\Pi\left(Q: \xi^{\prime}\right)$. Altogether, we obtain

$$
V(Q: \xi)=\langle\xi, \Phi\rangle=\left\langle\xi^{\prime}, \Phi\right\rangle\left\langle\left\langle\frac{\xi}{\left|\xi^{\prime}\right|}, \Phi\right\rangle=V\left(Q: \xi^{\prime}\right),\right.
$$

since $\left|\xi^{\prime}\right|<1$. This gives a projection into a $k$-plane $L\left(\xi^{\prime}\right) \subset W$ which is larger than $\Pi(Q: \xi)$, and completes the proof.

Proposition 2 has an interesting generalization even if $\operatorname{dim} Q=n$.

Definition. The Cartan subspace $C(\Phi), \Phi \in \Lambda_{k} R^{n}$, is the smallest subspace $W$ of $R^{n}$ such that $\Phi \in \Lambda_{k} W$.

A $k$-vector $\Phi$ is simple if and only if $\operatorname{dim} C(\Phi)=k$, in which case $C(\Phi)=L(\Phi)$. In general, the retraction theorem of E. Cartan [12] implies

$$
\left.C(\Phi)=\{\alpha\rfloor \Phi \mid \alpha \in \Lambda_{k-1} R^{n}\right\}
$$

Now, let $\Phi$ be a projection form of $Q$ for the $k$-planes in $\overline{\langle L\rangle}$, and assume $\operatorname{dim}(W=C(\Phi))<n$. We would like to conclude that the maximum of $V\left(Q: L^{\prime}\right)$, $L^{\prime} \in \overline{\langle L\rangle}$, occurs at a $k$-plane $L^{\prime} \subset W$. However, the proof in Proposition 2 requires that the form $\Phi$ gives the volume of projections of $Q$ into the $k$-planes of $\Pi(\overline{\langle L\rangle}: W)$. Thus we conclude

Corollary 3. If $\Phi$ is a projection form of $Q$ throughout the region $\overline{(L\rangle}$, and $\Pi(\overline{\langle L\rangle}: C(\Phi)) \subset \overline{\langle L\rangle}$, then the maximum of $V(Q: L)$ on $\overline{\langle L\rangle}$ is attained by a $k$-plane in $C(\Phi)$.

\section{Transforms}

The main result of this section is that certain Gale, c.s., and zonal transforms can be used to determine volume, as well as combinatorial type. This was already known for zonal transforms (see [21]). We begin with a common geometric description of the transforms which follows that in Section 3.1 of [24] (see also [22]). 
Let $Q \subset R^{n}$ be a convex polytope with $0 \in$ int $Q$, so that $0 \in \operatorname{relint} P=\Pi(Q: L)$, $L \in G(k, n)$. For convenience we will denote projection into $L$ in this section by $x^{\prime}=\Pi(x: L), x \in R^{n}$. The polar set of $P$ is the polytope

$$
P^{*}=\left\{y \in L \mid y \cdot x^{\prime} \leq 1, \forall x^{\prime} \in P\right\} .
$$

Alternatively, we can write

$$
P^{*}=\{y \in L \mid y \cdot x \leq 1, \forall x \in Q\}=Q^{*} \cap L,
$$

since $y \cdot x^{\prime}=y \cdot x$.

This polarity establishes an anti-isomorphism between the face-lattices of $P$ and $P^{*}$. If $F^{\prime} \in F(P)$, then the dual face $\left(F^{\prime}\right)^{*} \in F\left(P^{*}\right)$ is

$$
\left(F^{\prime}\right)^{*}=\left\{y \in P^{*} \mid y \cdot x^{\prime}=1, \forall x^{\prime} \in F^{\prime}\right\},
$$

(see 3.4 of [15]). It follows that

$$
\operatorname{relint}\left(F^{\prime}\right)^{*}=\left\{y \in P^{*} \mid y \cdot x^{\prime}=1, x^{\prime} \in F^{\prime} \text {, and } y \cdot x^{\prime}<1, x^{\prime} \notin F^{\prime}\right\},
$$

and that an arbitrary subset $F^{\prime} \subset P$ is a face of $P$ if and only if the set in (3.4) is nonempty. Letting $F=\Pi_{L}^{-1}\left(F^{\prime}\right) \cap Q$, this condition becomes: $F^{\prime} \subset P$ is a face of $P$ if and only if $\exists y \in P^{*}$ such that

$$
y \cdot x=1, \quad \forall x \in F, \quad \text { and } \quad y \cdot x<1, \quad \forall x \notin F .
$$

The latter statement is equivalent to

$$
\exists y \in L \cap \text { relint } F^{*},
$$

and also

$$
0 \in \operatorname{relint} \Pi\left(F^{*}: L^{\perp}\right) .
$$

This result implies that the combinatorial type of $\Pi(Q: L)$ can be determined from $\Pi\left(Q^{*}: L^{\perp}\right)$.

\section{Gale Transforms}

Let $P \subset R^{n}$ be a $k$-dimensional polytope with $n$ vertices and $0 \in$ relint $P$, and let $L=\operatorname{lin} P$. Then $P=\Pi(T: L)$ for some $(n-1)$-dimensional simplex $T$ which also contains 0 in its interior.

Given $\lambda \in \Lambda(n, r)$, the complementary sequence $\bar{\lambda} \in \Lambda(n, n-r)$ consists of $\{1, \ldots, n\} \backslash\left\{\lambda_{1}, \ldots, \lambda_{r}\right\}$. Label vert $T=\left\{y_{1}, \ldots, y_{n}\right\}$ and vert $T^{*}=\left\{z_{1}, \ldots, z_{n}\right\}$, so that for any $F_{\lambda}=\operatorname{conv}\left\{y_{\lambda_{1}}, \ldots, y_{\lambda_{r}}\right\} \in F(T)$, the dual face of $T^{*}$ is $F_{\lambda}^{*}=$ $\operatorname{conv}\left\{z_{\bar{\lambda}_{1}}, \ldots, z_{\bar{\lambda}_{n-r}}\right\}$. In the situation described here, (3.5) implies

$$
\Pi\left(F_{\lambda}: L\right) \in F(P) \Leftrightarrow 0 \in \operatorname{relint} \Pi\left(F_{\bar{\lambda}}^{*}: L^{\perp}\right) .
$$


The points $\Pi$ (vert $T^{*}: L^{\perp}$ ) are a Gale transform of $P$, and (3.6) becomes quite useful in obtaining combinatorial results when the codimension of $L$ is small (see [15] and [30]). However, here we are interested in the relationship of the transform to the volume of $P$.

First we must assume $P$ is in fact the projection of a regular $(n-1)$-simplex $T_{0} \subset R^{n-1}$, so that the dual simplex $T_{0}^{*}$ is $-\alpha T_{0}, \alpha>0$. In the next section we will show that an arbitrary polytope differs by a linear transformation from the projection of a regular simplex.

We now construct a $k$-vector $\Phi^{\perp} \in \Lambda_{n-k} R^{n-1}$ from the polytope $\Pi\left(T_{0}: L^{\perp}\right)$, with the property that

$$
V(P)=\left\langle\xi^{\perp}, \Phi^{\perp}\right\rangle
$$

where $\xi^{\perp}$ is an orientation of $L^{\perp}$.

Suppose $\xi$ is an orientation of $L$, and $\Phi$ is given by (2.3) so that $V(P)=\langle\xi, \Phi\rangle$. The volume of a typical pyramid is

$$
\frac{1}{k !}\left|\left\langle\xi, y_{\lambda}\right\rangle\right|=V\left(\operatorname{conv}\left\{0, F_{\lambda}^{\prime}\right\}\right)
$$

$\lambda \in \Lambda(n, k)$ and $F_{\lambda}^{\prime} \in \Gamma(\partial P)$. From (1.9) we obtain

$$
\left\langle\xi, y_{\lambda}\right\rangle=\left\langle{ }^{*} \xi,{ }^{*} y_{\lambda}\right\rangle
$$

Recall that (1.10) implies $L\left({ }^{*} y_{\lambda}\right)=\left(\operatorname{lin} F_{\lambda}\right)^{\perp}$, and that $\left.\right|^{*} y_{\lambda}|=| y_{\lambda} \mid$. However, since $T_{0}$ is regular, $\left(\operatorname{lin} F_{\lambda}\right)^{\perp}$ is parallel to aff $F_{\bar{\lambda}}$. Therefore, ${ }^{*} y_{\lambda}$ is a multiple of $f_{\bar{\lambda}}$, i.e.,

$$
{ }^{*} y_{\lambda}= \pm\left|y_{\lambda}\right| \frac{f_{\bar{\lambda}}}{\left|f_{\bar{\lambda}}\right|} \text {. }
$$

Equations (3.8) and (3.9) show that the $k$-vector

$$
\Phi^{\perp}=c \sum \operatorname{sign}\left(\left\langle\xi^{\perp}, f_{\bar{\lambda}}\right\rangle\right) f_{\bar{\lambda}}
$$

satisfies $(3.7)$, where

$$
c=\frac{(1 / k !)\left|y_{\lambda}\right|}{\left|f_{\lambda}\right|}=\frac{(1 / k) \sqrt{1 / k-1 / n} V\left(T^{k-1}\right)}{V\left(T^{n-k-1}\right)}
$$

and

$$
V\left(T^{n}\right)=\frac{\sqrt{n+1}}{n !}
$$

In general, the terms appearing in (3.10) depend upon the triangulation of $\partial P$, but if the projection is sharp, they are determined solely by the condition $0 \in$ relint $\Pi\left(F_{\tilde{\lambda}}: L^{\dot{4}}\right)$ 
The methods above carry over to the projections of any dual pair of regular polytopes. However, in five or more dimensions [8, Chapter VII], the only other regular polytopes are the cross polytope and the cube. Applying (3.5) to this dual pair gives the geometric formulation of c.s. transforms [25] and zonal transforms [23]. We shall describe here simply the transformation of the projection forms.

\section{C.s. Transforms}

Let $\left\{e_{1}, \ldots, e_{n}\right\}$ be the standard orthonormal basis of $R^{n}$. Then $X^{n}=$ $\operatorname{conv}\left\{ \pm e_{1}, \ldots, \pm e_{n}\right\}$ is a regular cross polytope. Let $P=\Pi\left(X^{n}: L\right), L \in G(k, n)$, be a centrally symmetric polytope with $2 n$ vertices. Construct a projection form $\Phi \in \Lambda_{\lambda} R^{n}$ by decomposing $P$ into pyramids with volume

$$
\frac{1}{k !}\left\langle\xi, e_{\lambda}\right\rangle=\frac{1}{k !}\left\langle{ }^{*} \xi{ }^{*} e_{\lambda}\right\rangle
$$

$\lambda \in \Lambda(n, k)$, and ${ }^{*} e_{\lambda}= \pm e_{\bar{\lambda}}$. Then the $k$-vector

$$
\Phi^{\perp}=\frac{1}{k !} \sum \operatorname{sign}\left(\left\langle\xi^{\perp}, e_{\bar{\lambda}}\right\rangle\right) e_{\bar{\lambda}}
$$

satisfies (3.7), where the sum again depends on $\Gamma(\partial P)$ if the projection is not sharp, and (3.5) otherwise.

\section{Zonal Transforms}

Since projection commutes with Minkowski sum, the projection of the cube $C^{n}=\sum\left[e_{i}\right]$ is the zonotope $Z=\sum\left[e_{i}^{\prime}\right], e_{i}^{\prime}=\Pi\left(e_{i}: L\right)$. According to Shephard [31], $Z$ can be decomposed into $\left(\begin{array}{l}n \\ k\end{array}\right)$ parallelotopes congruent to $\Sigma\left[e_{\lambda_{1}}^{\prime}\right], \lambda \in \Lambda(n, k)$. Thus the projection form of $Z$ is

$$
\Phi=\sum_{\Lambda(n, k)} \operatorname{sign}\left(\left\langle\xi, e_{\lambda}\right)\right) e_{\lambda}
$$

and, by $(3.11)$,

$$
\Phi^{\perp}=\sum_{1(n, n-k)} \operatorname{sign}\left(\left\langle\xi^{\perp}, e_{\lambda}\right\rangle\right) e_{\tilde{\lambda}}
$$

But this $k$-vector is also the projection form of $\Pi\left(C^{n}: L^{1}\right)$, and so (3.7) gives

$$
V\left(C^{n}: L\right)=V\left(C^{n}: L^{\perp}\right)
$$

(see also [22]). 


\section{Extremum Problems}

In the remainder of the paper we shall apply the machinery developed above to various extremum problems, chiefly that of maximizing the volume of the projection of a polytope.

A star $S=\left\{s_{1}, \ldots, s_{n}\right\}$ in $R^{k}$ is a finite set of vectors which span $R^{k}$. The $n \times k$ matrix whose $i$ th row contains the coordinates of $s_{i}$ will be denoted by $\mathbf{S}$. We denote the collection of all stars with $n$ vectors in $R^{k}$ by $S^{k}$. A star is said to be "eutactic" if it is the projection of an orthonormal frame in $R^{n}$, and the eutactic stars in $S^{k}$ will be denoted by $\Pi^{k}$. An application of the Gram-Schmidt process yields (see [16])

Theorem 4. A star $S$ is eutactic if and only if $\mathbf{S}^{\prime} \mathbf{S}=\mathbf{I}$.

For symmetrical stars, we have $[8,13.91]$

Theorem 5. A star $S$ is eutactic if its symmetry group is irreducible.

Thus, the vertices of any regular or uniform polytope [7] form a eutactic star. A recent discussion of eutactic stars appears in [29].

If $E=\left\{e_{1}, \ldots, e_{n}\right\}$ is the standard orthonormal basis of $R^{n}$, then the polytopes

$$
\begin{aligned}
T_{n-1} & =\operatorname{conv} E, \\
X^{n} & =\operatorname{conv}\{ \pm E\}, \\
C^{n} & =\sum\left[e_{i}\right]
\end{aligned}
$$

are a regular $(n-1)$-simplex, $n$-cross polytope, and unit $n$-cube, where the sum represents the Minkowski sum of the line segments $\left[e_{i}\right]=\operatorname{conv}\left\{-e_{i} / 2, e_{i} / 2\right\}$. Similar operations on the eutactic star $S=\Pi(E: L), L \in G(k, n)$, define projections of these figures.

A star $S$ is said to be "balanced" if $\sum s_{i}=0$. It follows that $S=\Pi(E: L)$ is balanced if and only if $L$ lies in the hyperplane through the origin parallel to aff $T^{n-1}$.

Choose a $k$-frame $X$ in $L$ so that the vectors of $X$ form an orthonormal set. The matrix equation for the coordinates (with respect to $X$ ) of the projection of a point $y \in R^{n}$ into $L$ is then

$$
\Pi(y: L)=(\mathbf{X} y)^{\prime},
$$

and therefore

$$
\mathbf{S}=\mathbf{I} \mathbf{X}^{\prime}=\mathbf{X}^{\prime}
$$

Theorem 4 implies that $\Pi^{k}$ is a subset of

$$
Y^{k}=\left\{\left.S \in S^{k}\left|\sum_{i=1}^{n}\right| s_{i}\right|^{2}=k\right\} .
$$


One last interesting family is the "spherical stars"

$$
\Omega^{k}=\left\{S \in S^{k}|| s_{i} \mid=\sqrt{k / n}, i=1, \ldots, n\right\}
$$

Henceforth the results shall be stated only for conv $S$, with the understanding that similar results also hold for conv $\{ \pm S\}$ and $\sum\left[s_{i}\right]$. In particular, the theorems for zonotopes can all be found in Sections 2 and 3 of [11].

Let $q: V(k, n) \rightarrow G(k, n)$ be the natural projection. We can associate a collection of stars

$$
S(L)=\left\{S \in S^{k} \mid S^{i}=\mathbf{X} \text { for some } X=q^{-1}(L)\right\}
$$

to any $L \in G(k, n)$. The remarks following (1.1) imply that $S(L)$ is the set of all stars linearly equivalent to $\Pi(E: L)$. The following theorem shows that maximizing the volume in $Y^{k}$ and $\Pi^{k}$ leads to the same figures.

Theorem 6. Conv $S$ attains its largest volume among stars in $Y^{k} \cap S(L), L \in$ $G(k, n)$, if and only if $S$ is eutactic.

Proof. Let $S \in Y^{k} \cap S(L)$. Then the $k$-frame $X$ given by $\mathbf{X}^{\prime}=S$ satisfies

$$
\sum_{i=1}^{k}\left|x_{i}\right|^{2}=\sum_{i=1}^{k} \sum_{j=1}^{n} x_{i j}^{2}=\sum_{j=1}^{n} \sum_{i=1}^{k} s_{i j}^{2}=\sum_{j=1}^{n}\left|s_{j}\right|^{2}=k
$$

If $S$ is eutactic, (2.2) implies that

$$
V(\operatorname{conv} S)=|\langle\xi, \Phi\rangle|
$$

where $\Phi \in \Lambda_{k} R^{n}$ is a projection form and $\xi=i(X)$. But if $f$ is a linear transformation of $L$, then $\left(\Lambda_{k} f\right)(\xi)=(\operatorname{det} f) \xi$ and $V(\operatorname{conv}(f(S))=(\operatorname{det} f) V(\operatorname{conv}(S))$. Hence, (4.6) holds for arbitrary stars, and can be rewritten

$$
V(\operatorname{conv} S)=|\xi|\left|\left\langle\frac{\xi}{|\xi|}, \Phi\right\rangle\right|
$$

Now, as $S$ varies in $S(L)$, the only term which changes in (4.7) is $|\xi|$. But, (1.5) implies

$$
|\xi|=V\left(\sum\left[x_{i}\right]\right)
$$

and given the condition in (4.5), it is easy to prove that this volume is maximized when the parallelotope $\sum\left[x_{i}\right]$ is a unit $k$-cube. In this case, $\mathbf{X} \mathbf{X}^{\prime}=\mathbf{S}^{\prime} \mathbf{S}=\mathbf{I}$, and thus $S$ is a eutactic star as required. 
An interesting corollary of this theorem follows from the fact that $\Omega^{k} \subset Y^{k}$.

Corollary 7. If a maximal projection in $\Pi^{k}$ is spherical, then it is also a solution to the problem of finding the largest volume of the convex hull of $n$ points on $a$ sphere in $R^{k}$.

The latter is a well-known isoperimetric problem (see [10]). In particular, the solution for five points on the 2-sphere is not eutactic [1], whereas the solutions in [10] and [18] are eutactic.

We now proceed to give two necessary conditions for these problems. The first condition can be proved exactly as in Lemma 1 of [2], which covers the case $k=3$. Let $\max Y^{k}$ denote the collection of stars $S \in Y^{k}$ such that $V(\operatorname{conv} S)$ has a relative maximum at $S$, and use a similar notation for the other families. If $P$ is a $k$-dimensional polytope, then the outer "area" normal to a facet $F_{\lambda} \in F_{k-1}(P)$, is the vector $n_{\lambda}$ which points away from int $P$ and satisfies $\left|n_{\lambda}\right|=V\left(F_{\lambda}\right)$.

Theorem 8. If $S \in \max Y^{k}\left(=\max \Pi^{k}\right)$ or $\max \Omega^{k}$, then $P=\operatorname{conv} S$ is simplicial, and, $\forall i \in 1, \ldots, n$,

$$
\frac{s_{i}}{\left|s_{i}\right|}=\frac{n_{i}}{\left|n_{i}\right|}
$$

where

$$
n_{i}=\left\{\sum n_{\lambda} \mid F_{\lambda} \in F_{k-1}(P) \text { and } s_{i} \in F_{\lambda}\right\}
$$

The analogous theorem for zonotopes states that maximal zonotopes are cubical, and (4.9) holds with

$$
n_{i}=\left\{\sum n_{\lambda} \mid n_{\lambda} \cdot s_{i}>0\right\}
$$

[11, Theorem 3.1].

We have seen in Section 1 that $\tilde{G}(k, n)$ is embedded as a submanifold of the unit sphere in $\Lambda_{k} R^{n}$. Given $\xi \in \tilde{G}(k, n)$ and a curve $\gamma: R \rightarrow \tilde{G}(k, n)$ with $\gamma(0)=\xi$, the $k$-vector

$$
\eta=\left.\gamma^{\prime}(t)\right|_{t=0}
$$

is called a tangent vector of $\tilde{G}(k, n)$ at $\xi$. The collection of all such tangent vectors at $\xi$ forms a subspace $T_{\xi} \tilde{G}(k, n)$ of dimension $k(n-k)$ in $\Lambda_{k} R^{n}$. The other necessary condition depends on the fact that the tangent plane $T_{\xi} \tilde{G}(k, n)$ is spanned by the simple vectors $t \wedge(e\rfloor \xi), \forall e \in \xi$ and $\forall t \perp e$ [17, Vol. II, p. 314].

Proposition 9. Given $\Phi \in \Lambda_{k} R^{n}$, the critical points of the function $\langle\xi, \Phi\rangle$ are those $\xi \in \tilde{G}(k, n)$ which satisfy

$$
(e\rfloor \xi)\rfloor \Phi=\alpha e
$$

$\forall e \in \xi$ and $\alpha \in R$. 
Proof. From the remark above, $\xi \in \tilde{G}(k, n)$ is a critical point if and only if

$$
\langle t \wedge(e \mid \xi), \Phi\rangle=0
$$

$\forall e \in \xi$ and $\forall t \perp e$. Substitution of (1.6) yields

$$
\langle t,(e\rfloor \xi)\rfloor \Phi\rangle=0
$$

which shows that $(e\rfloor \xi)\rfloor \Phi$ lies in the same direction as $e$.

Note that Proposition 9 applies only to sharp projections. It is not hard to show that if $Q=T^{n}$, the conditions in Theorem 8 and Proposition 9 are equivalent.

The problems of finding the largest projection of a polytope $Q$ in $R^{n}$ into $R$ or $R^{n-1}$ have simple solutions since the corresponding Grassmannians are isomorphic to the unit $(n-1)$-sphere. The largest projection into a line is just the maximum width. The largest projection into a hyperplane can be found by maximizing $\left|\sum n_{\lambda}\right|$ over all subsets of the facets of $Q$. This condition, along with many results for regular polytopes, can be found in [20].

\section{Projections into $R^{k}, 1<k<n-1$}

The "comass" of a $k$-vector $\Phi \in \Lambda_{k} R^{n}$ is defined to be

$$
\|\Phi\|=\{\max \langle\xi, \Phi\rangle \mid \xi \in \tilde{G}(k, n)\}
$$

(see 1.8 of [9]) and the face of $\tilde{G}(k, n)$ with supporting hyperplane orthogonal to $\Phi$ is

$$
G(\Phi)=\{\xi \in \tilde{G}(k, n) \mid\langle\xi, \Phi\rangle=\|\Phi\|\}
$$

If $\Phi$ is a projection form of a polytope $Q \subset R^{n}$, and $G(\Phi) \subset \overline{\chi(\Phi)}$ (see (2.6)), then $G(\Phi)$ equals the set of oriented $k$-planes $\xi$ for which $V(Q: \xi)=$ $\{\max V(Q: \eta) \mid \eta \in \overline{\chi(\Phi)}\}=\|\Phi\|$.

The classification of the faces of $\tilde{G}(2, n)$ (or $\tilde{G}(n-2, n)$ ) follows directly from the canonical representation of a 2 -form $\left[28\right.$, p. 2]. Given $\Phi \in \Lambda_{2} R^{n}$, there exists an orthonormal sequence $e_{1}, \ldots, e_{2 m} \in R^{n}$ and real numbers $\lambda_{1} \geq \lambda_{2} \geq \cdots \geq \lambda_{m}>$ 0 , such that

$$
\Phi=\sum_{j=1}^{m} \lambda_{j} e_{2 j-1} \wedge e_{2 j}
$$

[9, p. 29]. If $\lambda_{1}>\lambda_{2}, G(\Phi)=e_{1} \wedge e_{2}$, while if $\lambda_{1}=\cdots=\lambda_{r}>\lambda_{r+1}, G(\Phi)$ is the set of 2-planes which are complex lines in $R^{2 r}=\operatorname{lin}\left\{e_{1}, \ldots, e_{2 r}\right\}$ under the complex 
structure $e_{2 j}=i e_{2 j-1}, j=1, \ldots, r$. By ruling out the second possibility, we obtain below a uniqueness result for the maximum projection in each combinatorial region. The basic idea is that an infinite face must contain a plane orthogonal to some 2-faces of $Q$, in which case there exists a nearby sharp projection with greater volume.

Let $\max \Pi_{k}(Q)\left(\operatorname{Max} \Pi_{k}(Q)\right)$ denote the set of $\xi \in \tilde{G}(k, n)$ for which $V(Q: \xi)$ has a relative (absolute) maximum.

Theorem 10. If $\xi \in \max \Pi_{k}(Q)$, then $\Pi(Q: \xi)$ is sharp.

Proof. Suppose $0 \in$ int $Q$ and the projection $P=\Pi(Q: \xi)$ is not sharp. The discussion in Section 2 implies that $\xi$ must belong to an intersection of hyperplane sections of $G(k, n)$ which form the boundaries of sharp projection regions. Hence, there exists a path $\gamma: R \rightarrow G(k, n), \gamma(0)=\xi$, such that the projections

$$
P^{+}=\Pi\left(Q: \xi^{+}=\gamma(\varepsilon)\right) \quad \text { and } \quad P^{-}=\Pi\left(Q: \xi^{-}=\gamma(-\varepsilon)\right)
$$

are sharp for some $\varepsilon>0$.

Let $\Phi$ be a projection form for $P$ given by (2.3). We claim that the projection form of $\mathrm{P}^{+}$can be written as

$$
\Phi^{+}=\Phi_{0}^{+}+\Phi \quad \text { with } \quad\left\langle\eta, \Phi_{0}^{+}\right\rangle \geq 0, \quad \eta=\gamma^{\prime}\left(0^{+}\right)
$$

The form $\Phi$ defines a triangulation $\Gamma$ of $P$ into $k$-simplices with apex 0 and base in $\partial P$. Choose $\varepsilon$ small enough to that these simplices remain nondegenerate for $-\varepsilon \leq t \leq \varepsilon$. Then in $\xi^{+}, \Gamma$ becomes a star-polytope with 0 in its kernel. Therefore, we can define $\Phi_{0}^{+}$by triangulating the part of $P^{+}$not in $\Gamma$. Since $\left\langle\xi^{+}, \Phi_{0}^{+}\right\rangle \geq 0$ and $\left\langle\xi, \Phi_{0}^{+}\right\rangle=0,\left\langle\eta, \Phi_{0}^{+}\right\rangle$must be nonnegative, and this establishes (5.4).

Construct the form for $P^{-}$in a similar manner so that

$$
\Phi^{-}=\Phi_{0}^{-}+\Phi \quad \text { with }\left\langle-\eta, \Phi_{0}^{-}\right\rangle \geq 0
$$

Note that at least one of $\left\langle\eta, \Phi_{0}^{+}\right\rangle$or $\left\langle-\eta, \Phi_{0}^{-}\right\rangle$must be strictly positive since $P$ is not sharp.

Now, the hypothesis that $V(Q: \xi)$ has a relative maximum at $\xi$ implies

$$
0 \geq\left\langle\eta, \Phi^{+}\right\rangle=\left\langle\eta, \Phi_{0}^{+}+\Phi\right\rangle \text { and } 0 \geq\left\langle-\eta, \Phi^{-}\right\rangle=\left\langle\eta, \Phi_{0}^{-}+\Phi\right\rangle .
$$

Adding these equations gives

$$
0 \geq\left\langle\eta, \Phi_{0}^{+}-\Phi_{0}^{-}\right\rangle
$$

But the remark following (5.5) implies

$$
\left\langle\eta, \Phi_{0}^{+}-\Phi_{0}^{-}\right\rangle>0,
$$

which gives a contradiction. 
In preparation for the final theorem, we assemble a few properties of $\tilde{G}(2,4)$. This Grassmannian sits inside $\Lambda_{2} R^{4} \approx R^{6}$ as a four-dimensional manifold isomorphic to a product of 2-spheres. Let $\left\{e_{1}, e_{2}, e_{3}, e_{4}\right\}$ be an orthonormal basis for $R^{4}$, and give $R^{4}$ the complex structure $e_{2}=i e_{1}$, and $e_{4}=i e_{3}$. Then the 2-sphere

$$
\Theta=\left\{\xi \in \tilde{G}(2,4) \mid \xi=x \wedge i x, x \in R^{4}\right\}
$$

equals the real planes which are complex lines in $R^{4} \approx \mathrm{C}^{2}$. A simple 2-vector is said to be "complex" if it lies in $\Theta$.

Let $\xi \in \tilde{G}(2,4)$ and let $H=\{\eta \in \tilde{G}(2,4) \mid\langle\eta, \xi\rangle=0\}$. Then the set $H \cap \Theta$ equals $\pm \xi^{\perp}$ if $\xi$ is complex, and is a circle otherwise.

Theorem 11. If $k=2$ or $n-2$, then $\left|\operatorname{Max} \Pi_{k}(Q) \cap \chi(\Phi)\right| \leq 1$ for any sharp projection form $\Phi \in \Lambda_{k} R^{n}$.

Proof. The duality in (1.9) shows that we need only consider the case $k=2$. We claim that it also suffices to take $n=4$. Assume $\xi, \xi^{\prime} \in \operatorname{Max}_{k}(Q)$ both lay in some open region on $\tilde{G}(k, n)$. Then they would have the same property for the polytope $\Pi(Q: W), W=L(\xi) \oplus L\left(\xi^{\prime}\right)$. Since $\operatorname{dim} W \leq 4$, this would contradict the result for $\tilde{G}(2,3)$ or $\tilde{G}(2,4)$.

Now it follows directly from (5.3) that the only relative maxima of $\langle\xi$, $\Phi\rangle$, $\xi \in \tilde{G}(2,4)$, are the planes in $G(\Phi)$. Thus, if $|G(\Phi)|=1$ we are done. Otherwise, $G(\Phi)$ equals the complex lines $\Theta$, and we may assume $\Theta \cap \overline{\chi(\Phi)} \subset \operatorname{Max}_{k}(Q)$.

In this case, the previous theorem implies $\Theta \cap \partial \overline{\chi(\Phi)}=\varnothing$, which is possible only if $\Theta \subset \chi(\Phi)$. But, by the remarks above, any hyperplane section which bounds $\overline{\chi(\Phi)}$ intersects $\Theta$ in at least two points. This contradiction gives the desired result.

Any generalization of Theorem 11 to higher dimensions must confront the increasing complexity of the Grassmannians. In the particular case of $\tilde{G}(3,6)$, Morgan [28] has shown that there are only four types of faces: a singleton, a doubleton, and two types of infinite faces. Theorems 10 and 11 can again be used to rule out the infinite faces.

\section{References}

1. Berman, J. D., and K. Hanes, Volumes of polyhedra inscribed in the unit sphere in $E^{3}$, Math. Ann. 188 (1970), 78-84.

2. Bokowski, J., and B. Sturmfels, Oriented Matroids and Chirotopes-Problems of Geometric Realizability, Preprint, TH, Darmstadt, 1985.

3. Bokowski, J., and B. Sturmfels, On the coordinatization of oriented matroids, Discrete Comput. Geom. 1 (1986), 293-306.

4. Bonnesen, T., and W. Fenchel, Theorie der Konvexen Korper, Springer-Verlag, Berlin, 1934.

5. Busemann, H., G. Ewald, and G. C. Shephard, Convex bodies and convexity on Grassmann cones, Math. Ann. 151 (1963), 1-41.

6. Chakerian, G. D., and P. Filliman, The measures of the projection of a cube, Studia Sci. Math. Hungar. 21 (1986), 103-110. 
7. Coxeter, H. S. M., Wythoff's construction for uniform polytopes, Proc. London Math. Soc. (2) 38 (1935), 327-339.

8. Coxeter, H. S. M., Regular Polytopes, Dover, New York, 1973.

9. Federer, H., Geometric Measure Theory, Springer-Verlag, Berlin, 1969.

10. Fejes Tóth, L., Regular Figures, MacMillan, New York, 1964.

11. Filliman, $\mathbf{P}_{\text {, }}$ Extremum problems for zonotopes, Geom. Dedicata, to appear.

12. Gardner, R., Some applications of the retraction theorem in exterior algebra, J. Differential Geom. $2(1968), 25-31$.

13. Goodman, $J_{*}$, and $\mathbf{R}$. Pollack, Upper bounds for configurations and polytopes in $R^{d}$, Discrete Comput. Geom. 1 (1986), 219-227.

14. Griffiths, P., and J. Harris, Principles of Algebraic Geometry, Wiley, New York, 1978.

15. Grünbaum, B., Convex Polytopes, Wiley, London, 1967,

16. Hadwiger, H., Über ausgezeichnete Vektorsterne und reguläre Polytope, Comm. Math. Helv. 13 (1940), 90-107.

17. Hodge, W., and D. Pedoe, Methods of Algebraic Geometry, Cambridge University Press, London 1947.

18. Linhart, J., Volume and circumradius of simplicial polytopes, in Discrete Geometry and Convexity, $1985,97-105$.

19. Linhart, J., An upper bound for the intrinsic volumes of equilateral zonotopes, preprint.

20. Martini, H., and B. Weissbach, Zur besten Beleuchtung konvexer Polyeder, Beitr. Geom. Algebra $17(1984), 151-168$.

21. McMullen, P., Volumes of projections of unit cubes, Bull. London Math. Soc. 16 (1984), 278-280.

22. McMullen, P., Transforms, diagrams and representations, in Contributions to Geometry (Proc. of the Geometry-Symp., Siegen) J. Tolke and J. M. Wills, eds., Birkhauser, Basel, 1978.

23. McMullen, P., On zonotopes, Trans. Amer. Math. Soc. 159 (1971), 91-109.

24. McMullen, P., and G. C. Shephard, Convex Polytopes and the Upper Bound Conjecture, London Math. Soc. Lecture Notes Ser, 3, Cambridge University Press, Cambridge, 1971.

25. McMullen, P., and G. C. Shephard, Diagrams for centrally symmetric polytopes, Mathematika 15 (1968), 123-138.

26. McMullen, P., and G. C. Shephard, Polytopes with an axis of symmetry, Canad. J. Math. 22 (1970), 265-287.

27. Milnor, J., and J. Stasheff, Characteristic Classes, Princeton University Press, Princeton, NJ, 1974.

28. Morgan, F., The exterior algebra $\Lambda^{k} R^{\prime \prime}$ and area minimization, Linear Algebra Appl. 66 (1985), $1-28$.

29. Seidel, J. J., Eutactic stars, Colloquia Mathematica Societatis János Bolyai 18. Combinatorics, Keszthely, Hungary, 1976, 983-999.

30. Shephard, G. C., Sections and projections of convex polytopes, Mathematika 19 (1972), 144-162.

31. Shephard, G. C., Combinatorial properties of associated zonotopes, Canad. J. Math. 26 (1974), 302-321.

32. Spivak, M, A Comprehensive Introduction to Differential Geometry, Publish or Perish, Berkeley, CA, 1979.

Received March 30, 1987, and in revised form November 18, 1987. 